\title{
Representaciones sociales de los estudiantes sobre el trabajo docente*
}

\author{
Social Representations of Students on Teacher's Duty
}

\author{
Luisa Carlota Santana Gaitán ${ }^{* *}$ Edilberto Hernández Cano***
}

Para citar este artículo: Santana, L. C.; Hernández, E. (2014). Representaciones sociales de los estudiantes sobre el trabajo docente. Infancias Imágenes, 13(2), 47-55

Recibido: 30-Mayo-2014 / Aprobado: 28-agosto-2014

\section{Resumen}

Con el propósito de contribuir a la discusión y comprensión del hacer del docente, este artículo presenta los resultados de la investigación realizada con un grupo de estudiantes de la Facultad de Ciencias y Educación de la Universidad Distrital acerca de las representaciones sociales que han construido sobre el trabajo de los docentes. Para su abordaje se asumió el enfoque estructural de Jean Abric, que permite identificar su núcleo y su estructura. La investigación de carácter cualitativo e interpretativo empleó técnicas interrogativas y asociativas. El análisis de contenido del corpus textual arrojó un campo representacional amplio del trabajo docente, que da cuenta del sentido que se le otorga, del hacer que se le atribuye y del docente propiamente dicho. El núcleo de la representación social se centra en considerar al docente y su trabajo como portadores de valores éticos y morales desde los cuales se propende por la trasformación social.

Palabras clave: enfoque estructural, enseñanza-aprendizaje, contexto, problemática

\begin{abstract}
In order to contribute to the discussion and understanding of the teacher duty, this article presents results of research carried out with a group of students from the Distrital University's faculty of science and education about social representations that have built on the work of teachers. To address this, the structural approach of Jean Abric was assumed, allowing to identify its core and structure. The qualitative and interpretative research employed interrogative and associative techniques. Content analysis of the textual corpus, showed broad representational fields of teaching, which accounts for the sense that is given, the cause is attributed and teaching itself. The core of social representation focuses on the teacher, and considers their work as bearers of ethical and moral values from which it is prone by social transformation.
\end{abstract}

Keywords: structural approach, teaching-learning, context, problematic

* Investigación financiada por la actual Vicerrectoría de Investigación, Innovación, Creación, Extensión y Proyección Social; inició en junio del 2009 y finalizó en febrero del 2013. El libro producto de la investigación se presentó en la XXVII versión de la Feria del Libro de Bogotá.

** Magíster en Desarrollo Educativo y Social, UPN-CINDE. Candidata a doctora en Educación de la Universidad Santo Tomás. Directora grupo de investigación Ciudad y Educación. Docente de la Facultad de Ciencias y Educación de la Universidad Distrital Francisco José de Caldas. Correo: dcasantana@yahoo.es

*** Magíster en Investigación Social Interdisciplinaria; especialista en Gerencia de Proyectos Educativos; especialista en Socialización Política y Democracia. Integrante del grupo de investigación Ciudad y Educación. Docente de la Facultad de Ciencias y Educación de la Universidad Distrital Francisco José de Caldas. Correo electrónico: edilbertoh14@gmail.com 


\section{INTRODUCCIÓN}

Las dinámicas de las instituciones están condicionadas por los diferentes actores que hacen parte de ellas, situación que no le es ajena a la universidad. Por tanto, es necesario obtener un conocimiento amplio de ellos que posibilite su descripción y comprensión para que se constituyan en agentes de cambio y construcción social en beneficio de la comunidad, al ser la universidad una institución social a la que se le asignan nuevos retos, relacionados directamente con la producción de conocimiento y la formación de profesionales socialmente activos que agencien cambios científicos y tecnológicos.

La universidad como organización social educativa, en un contexto como el actual, de profundas transformaciones que afectan a todos los ámbitos y sectores de la sociedad, enfrenta escenarios de crisis que le exigen generar propuestas novedosas frente a sus problemáticas de rendimiento académico, deserción universitaria, desarrollo de la carrera (Misas, 2004; Rugarcía, 1999; Borrero, 1989), que generan cuestionamientos por parte de la sociedad sobre su quehacer y la consecuente demanda de calidad en la oferta de la enseñanza que esta institución realiza en la preparación de profesionales y en la formación de los jóvenes.

En este contexto, las investigaciones sobre el docente y su formación resultan significativas, al ser él uno de los ejes importantes en los procesos de transformación educativa (Tedesco, 2001), pero igualmente por reconocerse su influencia como el sujeto más importante, fuera del hogar, que afecta los aprendizajes y el desarrollo del educando (Sánchez, 2004; Eggen y Kauchak, 2001; Petrovsky, 1990).

Los estudios sobre el trabajo docente se han realizado mayoritariamente en niveles educativos diferentes al universitario, basados en la lectura que los investigadores hacen de él o en lo que los docentes dicen de su propio trabajo. Esta investigación, por su parte, permitió describir, analizar y comprender el trabajo de los docentes universitarios desde las representaciones sociales construidas por los estudiantes, y concentró el interés, por un lado, en develar su contenido, mediante el análisis de los significados, y, por el otro, en identificar su núcleo y periferia.

La indagación de las representaciones sociales (en adelante RS) como modos o formas de conocimiento y, a la vez, como procesos simbólicos relacionados con el mundo y el accionar del hombre en él posibilita la comprensión de los procesos educativos y las dinámicas institucionales, al ser fenómenos de interacción social profundamente influenciados por el contexto sociocultural (Casado y Calonge, 2000; Casado y Martínez, 2000).

Las RS que las personas tienen sobre los diferentes hechos de su entorno y de los actores que hacen parte de estos inciden en su forma de actuar, en las estrategias que emplean, en el papel que se atribuyen dentro de la interacción, en las prácticas cotidianas, en la forma como los sujetos se aproximan al conocimiento y reconocen o no su papel en su proceso de construcción (Cardoso, 2011; Alves-Mazzotti, 2008; Restrepo, 1987.

Es por ello que su conocimiento se considera relevante al momento de entender las problemáticas de las instituciones y proponer acciones de transformación para su solución y lograr así los propósitos relacionados con la formación, el proceso de enseñanza-aprendizaje y la construcción de saberes y conocimientos (Castorina, 2012).

\section{Sobre el contexto conceptual}

\section{Las representaciones sociales}

La teoría de las RS fue propuesta por Serge Moscovici en 1961, quien las define como:

Una modalidad particular de conocimiento, cuya función es la elaboración de los comportamientos y la comunicación entre los individuos; es un corpus organizado de conocimientos y una de las actividades síquicas, gracias a las cuales los 
hombres hacen inteligible la realidad física y social, se integran en un grupo o en una relación cotidiana de intercambios, liberando los poderes de la imaginación (p.150).

Para Jodelet (1986), discípula de Moscovici, las RS son una determinada manera de interpretar y pensar la realidad cotidiana de los individuos y grupos, que permite fijar su posición en relación con situaciones, objetos y comunicaciones que les compete; se constituyen en una forma de identificar a un grupo a través de sus propias miradas y perspectivas, para comprender los significados sociales que construyen y que le dan sentido a su existencia.

Después de los planteamientos de Moscovici, en el estudio de las RS cobra relevancia el enfoque estructural, conocido como "teoría de núcleo central", propuesto por Jean-Claude Abric (2001). Para el autor, las RS son a la vez producto y proceso de una actividad mental, por la que un individuo o un grupo reconstituyen la realidad y le atribuyen una significación específica, por tanto, no son un simple reflejo de la realidad, sino una organización significante.

Desde esta perspectiva, una RS se define por dos componentes: el contenido y la organización de ese contenido o estructura, que reposa sobre una jerarquía entre los elementos determinada por el núcleo central. La organización del contenido de las representaciones en un sistema central y un sistema periférico hace que al mismo tiempo sean estables y móviles, rígidas y flexibles, consensuadas pero también marcadas por diferencias entre los sujetos. Desde este enfoque y las metodologías que propone para el estudio de las RS se desarrolló esta investigación.

\section{El trabajo docente}

El trabajo docente, cuya representación por parte de los estudiantes se constituyó en el objeto de la investigación, ha sido abordado desde diferentes perspectivas. Una de ellas lo analiza desde las condiciones y variables relacionadas con su particularidad laboral como actividad económica, soslayando el análisis sobre su accionar pedagógico, destacando las funciones de la docencia, la cualificación y formación del docente, las características del docente propiamente dicho, las particularidades de su relación con los estudiantes y su identidad profesional.

Otra perspectiva, expresada en autores como Birgin y Dussel (2000), hace énfasis en la función de la enseñanza como eje central del trabajo docente, en el papel de la pedagogía como práctica reflexiva de las formas de transmisión cultural y en el docente como autoridad, desde el que se reconoce, por un lado, como anotan Cabrera y Jiménez (citados por las autoras, 2000), el carácter intelectual del trabajo docente inmerso en las relaciones sociales, los medios simbólicos y sus dinámicas, que no permite reducirlo a la esfera económica; y, por el otro, como precisa Tardif (2004), la importancia de los saberes del docente construidos desde la historia vital, su experiencia personal, profesional y pedagógica.

Por consiguiente, la multiplicidad de factores que se han sido considerados a la hora de hablar del trabajo docente arroja las siguientes categorías de análisis: 1) El factor académico: identifica el significado que se le atribuye al trabajo docente y las actividades que lo conforman; 2) el factor profesional: se refiere a la formación del docente y sus características, es decir, a los requerimientos para ejercer el trabajo docente, el reconocimiento de las condiciones en que este se ha venido desarroIlando en el ámbito universitario y las problemáticas que inciden en el desempeño de la profesión; 3) el factor personal: posibilita el reconocimiento de las características deseables y no deseables en los docentes, para el ejercicio de la docencia; y 4) el factor sociorrelacional: identifica las características en las relaciones que se establecen entre el docente y el estudiante, cómo se ve al estudiante dentro del trabajo docente y qué papel desempeña cada uno de los actores en el proceso de enseñanza-aprendizaje. 
Estas categorías se constituyen en el punto de partida de la indagación, para posteriormente profundizar en la categoría de análisis "trabajo docente" desde las construcciones discursivas de los estudiantes.

\section{Sobre la ruta}

La investigación asumió una perspectiva fundamentalmente cualitativa de corte interpretativo, que reconoce en el abordaje de las representaciones un acercamiento plurimetodológico para la indagación del contenido, la estructura y el núcleo de las RS, mediante el uso de métodos interrogativos, asociativos y analíticos, según la propuesta de Abric (2001).

Por un lado, el contenido se abordó como campo semántico, y en esa medida se develaron los significados construidos por los estudiantes con relación al trabajo de los docentes, lo que permitió analizar y situar el sistema de categorías utilizado por los sujetos; por el otro, se buscó extraer los elementos organizadores de ese contenido e identificar el núcleo de la representación, considerando la frecuencia de evocación de los términos con los cuales se asocia la expresión "trabajo docente", mediante la cuantificación de material cualitativo y la determinación de la frecuencia y el rango de aparición, teniendo en cuenta que la congruencia y la significación en los dos criterios es un indicador de la centralidad del elemento.

Como técnicas de recolección de información se utilizaron: el cuestionario, construido a partir de categorías preestablecidas definidas desde la revisión teórica; por su parte, los grupos de discusión, la carta asociativa y la asociación de palabras privilegiaron los datos emergentes. La información recolectada fue asumida como un corpus textual y sometida al análisis de contenido, ya que el interés de la investigación se centró en los contenidos expresados por los sujetos de investigación y por las interpretaciones a sus respuestas, de tal manera que se pudiera develar el sentido que ha tenido y tiene para los estudiantes las representaciones en torno al tema.

\section{RESULTADOS Y CONCLUSIONES}

El análisis interpretativo de la información permitió identificar tres categorías en las RS sobre el trabajo docente: representaciones que dotan de sentido el trabajo de los docentes, representaciones del hacer de los docentes y representaciones del docente, que conforman el contenido de las representaciones. Para finalizar, se presenta el análisis y la reflexión sobre la estructura y núcleo central de la representación social.

\section{Representaciones que dotan de sentido el trabajo de los docentes}

El significado que tiene el trabajo docente recobra en el contexto de esta investigación un carácter particularmente importante, ya que no solo da cuenta de la razón de ser que los estudiantes le atribuyen, sino que permite una prefiguración del sentido que tiene su formación como futuros docentes.

\subsection{Formación de sujetos}

Un elemento que tiene un significado preponderante para los estudiantes es el hecho de considerar que el trabajo docente está orientado a su formación como sujetos, entendida como la posibilidad de construir conciencia sobre la realidad en la que se vive, una postura crítica y reflexiva que les permita ejercer acciones de transformación y cambio social. Igualmente, la formación como sujetos se concibe desde una perspectiva que involucra todas las áreas de desarrollo del ser humano, formación que se concibe como integral y en la cual se resalta la importancia de la educación en valores. Entre estos valores el compromiso y la responsabilidad en su profesión, con los estudiantes y con la sociedad, se consideran muy importantes en el ejercicio del trabajo docente. Los estudiantes le otorgan al quehacer docente un alcance significativo al considerar que su accionar repercute no solo en sí mismos, sino también en las personas y en la sociedad en general. 
Es de destacar cómo esta representación de los estudiantes centra el trabajo de los docentes en una dimensión axiológica, más allá de la enseñanza de los saberes disciplinares específicos. El trabajo docente se representa como motor de transformación social, formador de la "masa crítica", de conciencia de la realidad y de desarrollo de valores como el compromiso y la responsabilidad con la sociedad.

\subsection{El docente como guía, apoyo y orientador}

El docente se constituye para los estudiantes en guía, orientador y acompañante de su proceso de formación en un sentido amplio e integral, es decir, en el desarrollo de sus diferentes áreas: personal, social y académica. Esta representación supera las concepciones del rol del docente centradas en perspectivas magistrocéntricas (maestro centro del proceso de enseñanza y aprendizaje) y lo sitúa como orientador y guía. Esto se traduce en apuestas pedagógicas donde el sujeto estudiante se asume como activo constructor de conocimiento y de su personalidad y como sujeto social acompañado en este proceso del apoyo del docente.

\subsection{El estudiante: razón de ser del trabajo docente}

Los estudiantes se consideran el eje, centro, fundamento y la razón del trabajo docente, sin el cual este no tendría sentido. Se conciben como sujetos en formación y se asumen como agentes activos en el proceso de enseñanza-aprendizaje, y desde este lugar se configuran como partícipes de la cualificación del trabajo de los maestros; es el sujeto estudiante el que instituye al sujeto docente, lo que da cuenta de una imagen positiva de sí mismo.

\section{Representaciones del hacer de los docentes}

Una de las formas de hacer visibles las representaciones sociales son las prácticas que desarrollan las personas en su cotidianidad, para este estudio la práctica docente, que refiere a todas aquellas actividades que forman parte del hacer de los docentes. Así entendido, las representaciones que dotan de sentido el trabajo docente se ven reflejadas en el hacer del docente, en su práctica. Por ello, los estudiantes consideran principalmente que el hacer del docente está centrado en la formación de sujetos críticos y reflexivos y en ser guías, orientadores y facilitadores de su proceso de formación integral. No se dejan de lado aspectos específicos, referidos a la enseñanza, el aprendizaje, los procesos curriculares y las problemáticas que lo afectan, cuando los estudiantes se refieren a los docentes de sus proyectos curriculares.

\subsection{La enseñanza: el deber ser de la docencia}

La enseñanza se constituye en la principal actividad que los estudiantes le asignan al trabajo de los docentes, considerada por diversos autores como la esencia de la docencia. Si bien para los estudiantes de la Facultad de Ciencias y Educación de la Universidad Distrital lo prioritario del accionar del docente es su formación como sujetos y orientadores de su proceso de formación, al referirse a los docentes de sus proyectos curriculares consideran que uno de los aspectos esenciales en el hacer es la enseñanza de conocimientos disciplinares. Expresan que lo que enseñan los docentes debe ser significativo y permitir la relación de los contenidos con la realidad.

\subsection{Planificación de la enseñanza}

Para los estudiantes, una de las actividades del trabajo docente está relacionada con los procesos curriculares, su planificación y desarrollo, concepción sistemática de la tarea docente que invita a superar las perspectivas contingentes en el desarrollo del proceso de enseñanza y aprendizaje, que se traduce en la improvisación en la consecución de los objetivos educativos. 


\subsection{La investigación en el transcurrir del hacer docente}

Una de las actividades que se considera debe realizar el docente es la investigación, sin embargo no le atribuyen una importancia significativa en el hacer del docente ni la ven como referente de relevancia en su educación, ya que la prioridad es asignada a la formación ética, al saber disciplinar y pedagógico. Esta representación se constituye en un llamado a las instituciones formadoras de maestros para que consideren la investigación como un eje central en el proceso de formación de los docentes, al ser ella la base de la producción del conocimiento y una estrategia activa y productiva en el ejercicio docente.

\subsection{Las problemáticas del trabajo docente}

Las RS del trabajo docente no dejan de lado las condiciones en las cuales se lleva a cabo. Los estudiantes las ponen de manifiesto y las concretan en problemáticas de diferente índole que involucran tanto el contexto general como sus propias características y las de los estudiantes. El trabajo docente se desarrolla en un contexto en el que confluye una multiplicidad de problemáticas de orden cultural, social, familiar y personal, que han sido vivenciadas por los estudiantes desde su rol, pero también en las diferentes formas de aproximarse a la escuela. El conocimiento de estas condiciones hace que el trabajo docente se represente como difícil y la vocación, necesaria para superar las dificultades, a las que se agrega una imagen subvalorada de la profesión docente y unas políticas educativas y laborales adversas.

\section{Representaciones del docente}

Las representaciones del sentido y del hacer del trabajo de los docentes se concretan en la figura del docente como persona, que es representada por los estudiantes desde una visión que involucra aspectos individuales, cognitivos y sociales, que inciden en su proceso de formación y en la construcción de una relación en beneficio del proceso de enseñanza y aprendizaje.

\subsection{Sobre la formación del docente}

La formación del docente es visualizada por los estudiantes como una necesidad que va más allá del campo disciplinar, que si bien es importante, necesita de la pedagogía como saber fundamental. El docente requiere de unos saberes y conocimientos que involucren la realidad social, política y cultural del país, la educación, los aspectos psicológicos y sociológicos de sus estudiantes, que le permitan en su hacer contextualizar los contenidos que enseña y considerar las particularidades psicosociales de los estudiantes.

Los estudiantes manifiestan que el docente debe en primer lugar poseer unos valores éticos y correspondientemente desarrollar su trabajo e interacción con ellos.

\subsection{El docente}

Los estudiantes se representan al docente como un sujeto portador y reflejo de valores como el respeto, la dedicación, el compromiso, la responsabilidad, la vocación, la entrega y el esfuerzo, expresados en sus actitudes. Igualmente al docente se lo representa como poseedor de saberes y conocimientos, relacionado con el hecho de considerar la enseñanza como actividad predominante en la práctica docente.

\subsubsection{Generador de ambientes}

Dentro de las características que destacan en un buen docente están las relacionadas con aspectos personales que favorecen su relación cercana con el estudiante y la generación de un ambiente que facilite la enseñanza, el aprendizaje y su formación integral. Los estudiantes señalan una amplia gama de características sociorrelacionales en los docentes, pero destacan el hecho de que 
sean incluyentes, abiertos, allegados a ellos y amables.

Lejos de las visiones que veían en el docente una autoridad, a la que se le obedecía desde una relación asimétrica con el estudiante y la construcción de un vínculo pedagógico centrado en el adulto, en los estudiantes de la Universidad Distrital se encuentra una perspectiva de la relación que si bien no se explicita como horizontal, sí se configura como tal en la medida en que se le concibe basada en el respeto y en la generación de un ambiente que facilita sus intervenciones.

Para los estudiantes, además del respeto, el reconocimiento que el docente tenga de cada uno es fundamental en el proceso de enseñanza-aprendizaje: sus saberes, sus estilos, su capacidad de crítica y el aporte que puedan realizar al trabajo docente, como ya se hizo explícito al abordar las representaciones del sentido del trabajo docente; además, es importante que los reconozcan por su nombre, sus contextos, problemáticas e intereses.

Tal representación es una exigencia a la concepción de la interacción pedagógica como una relación interpersonal que implica el conocimiento y entendimiento mutuo de los actores involucrados, lo cual desemboca en un proceso pedagógico y didáctico pertinente a las necesidades, intereses y demandas de los educandos.

\subsubsection{Las características personales también cuentan}

En la representación social que del docente tienen los estudiantes, sus características personales son muy importantes como potenciadoras y generadoras de interacciones sociales, que facilitan el aprendizaje y la enseñanza.

Es de anotar que la responsabilidad y el compromiso fueron señalados como pilares fundamentales en la representación del sentido del trabajo docente, al igual que la formación crítica y reflexiva; en correspondencia con ello, al docente se le asume como sujeto crítico y reflexivo, que además requiere de creatividad y dinamismo.
Es importante señalar que los estudiantes también plantean las características que no son deseables en el docente, relacionadas con su personalidad, como son: el autoritarismo, la pereza, la "pedantería y prepotencia", el mal carácter; y con la práctica: la mediocridad, la monotonía, la arrogancia y la indiferencia son las más destacadas. Estas características no solo perjudican el desarrollo de trabajo, sino que agudizan la imagen negativa que presenta la docencia y su ejercicio.

El docente en su práctica aporta a la interacción sus propios marcos de referencia: personales, sus maneras de ser, sus necesidades e intereses que pueden convertirse en facilitadores u obstaculizadores de los propósitos educativos.

\section{Sobre la organización y el núcleo central de la representación social del trabajo de los docentes}

Los distintos análisis permiten concluir que el núcleo de la representación del trabajo de los docentes está en que es considerado una actividad social portadora de valores éticos y morales, que se reflejan en la responsabilidad y compromiso del docente con su labor, con los estudiantes y con la sociedad. Tanto el trabajo del docente como él y su función principal con los estudiantes está en la formación de sujetos éticos que mediante la reflexión y la crítica aportan a la transformación de la realidad. En este proceso, el docente es principalmente un guía y orientador de la formación personal y académica de los estudiantes.

Por consiguiente, la labor de enseñar, significativa dentro del hacer del docente, no se centra únicamente en la transmisión de saberes y conocimientos, sino que también debe promover la formación integral de los estudiantes. En este proceso se considera fundamental la construcción de una relación orientada por los valores, dentro de los cuales se destaca el respeto por el otro y por el sujeto en formación, el estudiante.

Las demás categorías expresadas en los resultados dan cuenta de los elementos periféricos de la 
representación, cuyo orden expresa la proximidad o no con el núcleo central. Las categorías próximas al núcleo desempeñan un papel importante en la concreción del significado de la representación; cuando están más distantes de él ilustran, aclaran, justifican esta significación (Abric, 2001).

\section{CONCLUSIONESY RECOMENDACIONES}

Los resultados arrojados dan cuenta de una representación del trabajo docente con una alta significación y valor para los estudiantes, en la medida en que repercute en su formación y desarrollo, en las diferentes áreas que constituyen al ser humano.

El trabajo docente es reconocido por los estudiantes a través su accionar principalmente ético, lo que implica la necesidad de una mayor comprensión por parte del docente de la valoración que en este sentido tiene su trabajo, no solo para reafirmarlo sino, ante todo, para iniciar procesos de transformación personal cuando su hacer no corresponde con las expectativas del estudiante y las demandas de la sociedad en términos de formación.

Los resultados de esta investigación arrojan un campo representacional amplio del trabajo docente, que abarcan el sentido del mismo, su hacer y al docente.

En cuanto a las representaciones que dotan de sentido el trabajo de los docentes, la formación integral del estudiante como sujeto connota una responsabilidad de su hacer que trasciende la formación en una disciplina específica, para repercutir en la vida de los estudiantes y en las posibilidades de su construcción como transformadores sociales.

Las representaciones del hacer del docente dejan entrever una visión amplia del accionar docente, centrado en la enseñanza relacionada con la realidad en la que se circunscriben los estudiantes.

Una de las actividades fundamentales del trabajo docente está relacionada con los procesos curriculares, su planificación y desarrollo, con lo cual se recomienda como parte de su hacer el aprendizaje de la construcción curricular, que involucra la determinación de los contenidos, su organización con base en los contextos y características cognitivas de los estudiantes, y, dentro de este proceso, la generación de las mejores condiciones que posibiliten la comprensión y movilización del saber resultante de la enseñanza y el aprendizaje.

En las representaciones del docente, su formación ética es el primer aspecto contemplado en el trabajo docente, y en relación con ella, los estudiantes se representan al docente como un sujeto portador y ejemplo de valores, con unas características personales que orientan su trabajo y la relación con los estudiantes, y que permiten una interacción cercana que contribuye a su formación integral.

El reconocimiento de la importancia del saber disciplinar y pedagógico es el segundo referente en la formación docente, donde se da prioridad a lo pedagógico sobre el conocimiento científico disciplinar. Esto permite ubicar a la pedagogía como el eje articulador de los demás tipos de saberes en la formación de los licenciados, ya que el dominio de un saber específico de conocimiento no es suficiente para ser enseñado.

En el trabajo docente, las características personales de los profesores condicionan las particularidades de las interacciones entre docente-estudiante, pues permiten conseguir o no la participación de los educandos en su proceso de formación y atender a sus distintas necesidades.

Los resultados y conclusiones de esta investigación dan cuenta del valor que tiene la indagación sobre las representaciones sociales en el contexto de la educación universitaria, en el logro de la comprensión de sus dinámicas y actores. Desde sus hallazgos facilita proponer procesos de cambio institucional, orientados a cumplir con las expectativas que tanto sus integrantes como la sociedad en general tienen de ella. Igualmente, se constituyen en un insumo importante en los procesos de reflexión personal deseables en la formación de los docentes, más aún en el caso de una Facultad de Educación, responsable de la formación de los futuros docentes. 


\section{REFERENCIAS}

Abric, J. (2001). Prácticas sociales y representaciones. México: Ediciones Coyoacán.

Alves-Mazzotti, A. (2008). Representações sociais: aspectos teóricos e aplicações à educação. Revista Múltiplas Leituras, 1(1), 18-43.

Birgin, A. y Dussel, I. (2000). Aportes para el abordaje curricular. Seminario: rol y trabajo docente. Secretaría de Educación. Buenos Aires. Recuperado de http://www.opech.cl/bibliografico/Doc Docente/S Rol y trabajo docente Birgin-Dussel.pdf

Borrero, A. (1989). Modelos universitarios y el modelo de la universidad latinoamericana. ¿Qué universidad para qué sociedad? Colección de Investigaciones, 1. Bogotá: UDFJC.

Cardoso, J. (2011). Contenido y estructura de representaciones sociales sobre pedagogía y pedagogos en profesores de ciencias (Tesis doctoral, Universidad de Burgos, España). Recuperado de http://dialnet.unirioja.es/servlet/ tesis? codigo $=23934$

Casado, E. y Calonge, S. (2000). Psicología social y educación. En E. Casado y S. Calonge. Representaciones sociales y educación (7-11). Caracas: Universidad Central de Venezuela.

Casado, E. y Martínez, C. (2000). Contenido, estructura y anclaje de la representación social en la interacción pedagógica. En E. Casado y S. Calonge. Representaciones Sociales y
Educación (35-58). Caracas: Universidad Central de Venezuela.

Castorina, J. (2012) Los usos del concepto de representación social en educación. Conferencia del Doctorado Interinstitucional en Educación. Bogotá: UDFJC.

Eggen, P. y Kauchak, D. (2001). Estrategias docentes. México: Fondo de Cultura Económica.

Jodelet, D. (1986). La representación social: fenómenos, concepto y teoría. En S. Moscovici (Comp.). Psicología social II. Barcelona: Paidós.

Moscovici, S. (1961). El psicoanálisis, su imagen y su público. Buenos Aires: Huemul.

Misas, G. (2004) La educación superior en Colombia: análisis y estrategias para su desarrollo. Bogotá: Universidad Nacional de Colombia.

Petrovsky, A. (1990) Psicología evolutiva y pedagógica. Moscú: Progreso.

Restrepo, M. (1987). Apuntes para una semántica de lo cotidiano. Signo y Pensamiento, 11.

Rugarcía, A. (1999). Hacia el mejoramiento de la educación universitaria. México: Trillas.

Sánchez, P. (2004). El proceso de enseñanza y aprendizaje. Madrid: Universidad Complutense.

Tardif, M. (2004). Los saberes del docente y su desarrollo profesional. Madrid: Narcea.

Tedesco, J. (2001). Los nuevos desafíos en la formación docente. En S. Sandoval (Comp.). La formación de educadores en Colombia. Tomo I. Bogotá: UPN.

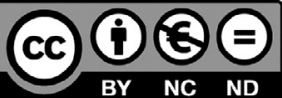

